\title{
THE ADULTEROUS TEXT
}

\section{PROLOGUE}

